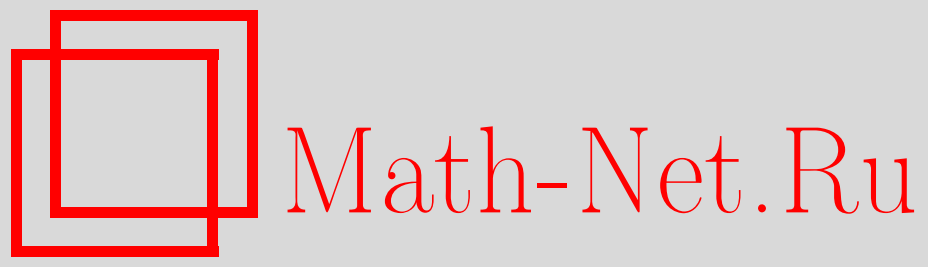

В. П. Маслов, Нелинейное финансовое осреднение, эволюционный процесс и законы эконофизики, Теория вероятн. и ее примен., 2004, том 49, выпуск 2, 269-296

DOI: https://doi.org/10.4213/tvp219

Использование Общероссийского математического портала MathNet.Ru подразумевает, что вы прочитали и согласны с пользовательским соглашением

http://www . mathnet.ru/rus/agreement

Параметры загрузки:

IP: 34.239 .49 .27

26 апреля 2023 г., 14:33:29

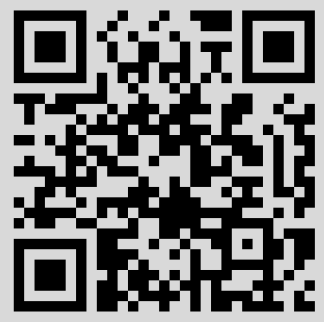




\section{НЕЛИНЕЙНОЕ ФИНАНСОВОЕ ОСРЕДНЕНИЕ, ЭВОЛЮЦИОННЫЙ ПРОЦЕСС И ЗАКОНЫ ЭКОНОФИЗИКИ}

Сходство и различие как методов, так и результатов финансовой математики и квантовой статистики могут быть полезны для той и другой науки. Рассмотрен процесс, пределом которого являются распределение Гиббса и финансовое осреднение; изучены законы эконофизики для покупателей, приводящие к «фазовым переходам» типа пробоя курса акций и дефолта; получены распределения Парето и Гаусса для доходов и расходов различных слоев обшества; введены понятия скорости оборота капитала для квазистабильного состояния общества.

Ключевые слова и фразы: эволюционный процесс, эконофизика, распределение Парето, сокращение информации, акции, финансы.

1. «Эконофизика». Аксиоматика финансового осреднения приводит в случае квазистабильного, осредненного рынка к некоторой формуле выигрыша-проигрыша, если задана случайная величина $\lambda$, принимающая значения $\lambda_{1}, \ldots, \lambda_{n}$, означающие, например, цены. Но в обычной частотной теории вероятностей выигрыш (проигрыш) вычисляется как математическое ожидание, умноженное на число испытаний:

$$
\sum_{i=1}^{n} g_{i} \lambda_{i}
$$

где $g_{i} / \sum_{j=1}^{n} g_{j}$ - частотные вероятности.

Спрашивается, какой достаточно естественный процесс в пределе приводит нас к финансовому осреднению? В силу того, что оно связано со значением свободной энергии в квантовой статистике, естественно спросить, какой процесс переводит произвольное распределение частиц по уровням энергии $\lambda_{i}$, полученное, например, с помошью «подсветки» материала,

$$
\frac{g_{i}}{\sum_{j=1}^{n} g_{j}}
$$

* Московский государственный университет им. М.В. Ломоносова, физический факультет, кафедра квантовой статистики и теории поля, Ленинские горы, 119992 Москва, Россия; e-mail: viktor_maslov@hotmail.com 
в распределение Гиббса. Разница с финансовым осреднением заключается только в знаке: это как бы свободная энергия с отрицательной температурой.

Однако если мы будем рассматривать не доход продавцов, а расход покупателей, то знак остается тот же, что и в распределении Гиббса, т.е. мы получим аналогию с квантовой статистикой при положительной температуре.

При определении искомого процесса мы; разумеется, должны применить некоторые способы осреднения, в том числе сокрашение информации - это также один из способов осреднения. Действительно, пусть имеется шестигранная кость с одинаковыми гранями и выигрышем, равным значениям $\lambda_{1}, \ldots, \lambda_{6}$, и пусть $\lambda_{2}=\cdots=\lambda_{6} \equiv \lambda$. Сократим игру, взяв монетку и поставив на одной ее стороне $\frac{5}{3} \lambda$, а на другой $\frac{1}{3} \lambda_{1}$. Это есть элементарное сокращение «информации» (см. [1], [3], [2, раздел 4]), учет равновероятности элементарных событий и некоторое «осреднение», так как вероятность есть осреднение частотных вероятностей.

Если осреднять по ансамблю независимых испытаний, то, как известно, единственной функцией от гамильтониана, которая позволяет это совершить, является экспонента от гамильтониана. В частности, эти наводящие соображения приводят нас к той формуле эволюции распределения (2), которая будет приведена ниже.

Мы будем рассматривать исходную случайную величину $\lambda$, принимающую значения $\lambda_{1}, \ldots, \lambda_{n}, \lambda_{i}>0$ (для простоты будем считать их несоизмеримыми).

Пусть $p_{i}$ - отвечаюшие им эмпирические вероятности, т.е. при $M$ испытаниях значение $\lambda_{i}$ выпадает $g_{i}$ раз,

$$
\sum_{i=1}^{n} g_{i}=M, \quad p_{i}=\frac{g_{i}}{M} .
$$

Сопоставим $\lambda$ случайную величину $Y$ по следующему правилу:

$$
Y_{N, K_{i}}=\sum_{j=1}^{n} \lambda_{i} K_{j} ; \quad \sum_{i=1}^{n} K_{i}=N .
$$

Случайная величина $Y_{N, K_{i}}$ отвечает исходу (выигрышу-проигрышу) в элементарной игре, например, при $n=2-$ в игре в «орлянку».

Вероятности соответствуюших выигрышей, как известно, равны (с точностью до нормировки)

$$
\frac{N !}{\prod_{i=1}^{n} K_{i} !} \prod_{i=1}^{n} g_{i}^{K_{i}}
$$

Рассмотрим схему сопоставления вероятностной задаче гильбертова пространства и оператора. Итак, случайная величина $\lambda$ имеет $n$ значений $\lambda_{1}, \ldots, \lambda_{n}$, соответствующие вероятности имеют вид $g_{i}$, а оператор $\widehat{H}_{1}$ задается матрицей размера $n M \times n M$, у которой на главной 
диагонали стоят $g_{1}$ раз числа $\lambda_{1}, g_{2}$ раз числа $\lambda_{2}, \ldots, g_{n}$ раз числа $\lambda_{n}$, а остальные элементы равны 0, таким образом, кратность собственного значения $\lambda_{i}$ равна $g_{i}$. Обозначим $l_{k}, k=1, \ldots, n$, его собственные векторы. Гильбертово пространство, отвечающее серии из $N$ испытаний, в этом случае есть $C^{n N M}$ - пространство векторов длины $N$, а гамильтониан $\widehat{H}_{N}$ выражается через $\widehat{H}_{1}$ по формуле

$$
\widehat{H}_{N}=\sum_{s=1}^{N} \widehat{E} \otimes \cdots \otimes \widehat{E} \otimes \widehat{H}_{1} \otimes \widehat{E} \otimes \cdots \otimes E,
$$

где $\widehat{E}$ - единичный оператор в пространстве $C^{n M}$, каждое слагаемое представляет собой тензорное произведение $N-1$ операторов $\widehat{E}$ и оператора $\widehat{H}_{1}$, причем в слагаемом с номером $s$ оператор $\widehat{H}_{1}$ в произведении стоит на $s$-м месте. Собственные векторы оператора $\widehat{H}_{N}$ имеют вид

$$
f_{k_{1}, \ldots, k_{N}}=e_{k_{1}} \otimes \cdots \otimes e_{k_{N}}
$$

где $k_{i}=1, \ldots, n$, а соответствующие собственные значения суть

$$
Y_{k_{1}, \ldots, k_{N}}=\lambda_{k_{1}}+\cdots+\lambda_{k_{N}} .
$$

Кратности собственных значений оператора $\widehat{H}_{N}$ совпадают с вероятностями «выигрыша» (5).

Рассмотрим теперь обобщения вероятностной задачи о выигрыше в серии из $N$ независимых испытаний. Рассмотрим выигрыш в серии из $N$ испытаний, причем объединим в одну серию все серии, у которых совпадают выигрыши (см. [4, гл. I, § 1, пример 6]), а различие состоит в порядке выпадения исходов $\lambda_{1}, \ldots, \lambda_{n}$. В некотором смысле можно считать, что испытания тождественны и от их перестановки ничего не меняется.

Сопоставим этой новой задаче пространство $C_{\mathrm{S}}^{n N M}$, которое является подпространством пространства $C^{n N M}$ и состоит из его элементов, имеющих вид

$$
f=\sum_{m_{1}=1}^{n} \cdots \sum_{m_{N}=1}^{n} f\left(m_{1}, \ldots, m_{N}\right) e_{m_{1}} \otimes \cdots \otimes e_{m_{N}}
$$

где $f\left(m_{1}, \ldots, m_{N}\right)$ - числовые коэффициенты, симметричные относительно перестановок $m_{s}$ и $m_{r}, s, r=1, \ldots, N, e_{m}$ - собственные векторы оператора $\widehat{H}_{1}$. Пространство $C_{\mathrm{S}}^{n N M}$ будем называть симметричным подпространством пространства $C^{n N M}$. Соответствующий оператор есть ограничение введенного выше оператора $\widehat{H}_{N}$ на подпространство $C_{\mathrm{S}}^{n N}$. Легко убедиться, что собственные значения этого оператора совпадают со значениями выигрыша, а их кратности равны

$$
\prod \frac{\left(g_{j}+K_{j}+1\right) !}{\left(g_{j}-1\right) ! K_{j} !}
$$


Рассмотрим еще одну игру в серии из $N$ испытаний. Так же как в предыдущей задаче, будем считать, что серии с одинаковым выигрышем, отличаюшиеся только перестановкой исходов $\lambda_{m_{1}}, \ldots, \lambda_{m_{N}}$, $m_{j}=1, \ldots, n$, объединяются в одну серию, а кроме того, наложим запрет на серии, в которых хотя бы один из исходов $\lambda_{1}, \ldots, \lambda_{n}$ встречается больше одного раза. Пространством, отвечаюшим такой постановке вероятностной задачи о выигрыше в серии из $N$ испытаний, является $C_{\mathrm{A}}^{n N M}$ - подпространство пространства $C^{n N M}$, состоящее из его элементов, представимых в виде (9), где коэффициенты $f\left(m_{1}, \ldots, m_{N}\right)$ антисимметричны относительно перестановок $m_{s}$ и $m_{r}, s, r=1, \ldots, N$. Будем называть $C_{\mathrm{A}}^{n N M}$ антисимметричным подпространством пространства $C^{n N M}$. Оператором в данном случае является ограничение оператора $\widehat{H}_{N}$ на подпространство $C_{\mathrm{A}}^{n N M}$. Так же как и в предыдуших случаях, собственные значения оператора совпадают со значениями выигрыша в этой новой игре, а их кратности, совпадаюшие с точностью до нормировки с вероятностями «выигрыша», равны

$$
\prod \frac{g_{j} !}{K_{j} !\left(g_{j}-K_{j}\right) !} \text {. }
$$

Рассмотренные задачи о серии испытаний принципиально отличаются одна от другой: в рассмотренных задачах существует различие между собственными функциями оператора $\widehat{H}_{N}$. Собственные функции гамильтониана стандартной задачи о выигрыше обладают следуюшим свойством: если представить их в виде столбца, состоящего из $n N M$ элементов, то квадрат каждого элемента равен сам себе, поскольку в такой записи векторы представляют собой столбцы из 0 и 1 . Поэтому в обычной задаче о серии независимых испытаний полуплотность (корень из плотности) совпадает с плотностью вероятности. Это означает, что мы можем ввести норму, отвечающую банахову пространству $L_{1}$, что мы и сделаем в следующем пункте.

2. Эволюционный процесс, приводящий к распределению Гиббса. Рассмотрим случайную величину $\lambda$, которая принимает $l$ различных значений: $\lambda_{1}, \ldots, \lambda_{l}$. Далее будем считать, что значения случайной величины находятся в общем положении, значит, для них имеет место условие: если для целых чисел $k_{1}, \ldots, k_{l}$ выполняются равенства

$$
\sum_{i=1}^{l} k_{i}=0, \quad \sum_{i=1}^{l} \lambda_{i} k_{i}=0
$$

то $k_{i}=0$ при всех $i=1, \ldots, l$.

Вновь сопоставим рассматриваемой случайной величине пространство $L$, размерность которого равна $n$, и оператор $\widehat{H}_{1}$ в этом пространстве. Оператор $\widehat{H}_{1}$ имеет набор собственных векторов $\mathbf{e}_{i}, i=1, \ldots, l$, образующих ортонормированный базис в пространстве $L$. Собственное 
значение, отвечающее собственному вектору $\mathbf{e}_{i}$, равно $\lambda_{i}$. Рассмотрим теперь серию из $M$ одинаковых испытаний, в каждом из которых результатом является выпадение одного из значений $\lambda_{1}, \ldots, \lambda_{l}$ случайной величины. Этой вероятностной задаче, согласно вышесказанному, соответствует пространство $\mathscr{L}_{M}$, которое является тензорным произведением $M$ пространств $L$. Векторы пространства $\mathscr{L}_{M}$ имеют вид

$$
\Psi=\sum_{i_{1}=1}^{l} \cdots \sum_{i_{M}=1}^{l} \psi\left(i_{1}, \ldots, i_{M}\right) \mathbf{e}_{i_{1}} \otimes \cdots \otimes \mathbf{e}_{i_{M}}
$$

где $\psi\left(i_{1}, \ldots, i_{M}\right)$ - произвольная функция набора дискретных переменных $i_{s}=1, \ldots, l, s=1, \ldots, M$.

Введем норму в пространстве $\mathscr{L}_{M}$ следуюшим образом:

$$
\|\Psi\|=\sum_{i_{1}=1}^{l} \cdots \sum_{i_{M}=1}^{l}\left|\psi\left(i_{1}, \ldots, i_{M}\right)\right| .
$$

Кроме пространства $\mathscr{L}_{M}$, серии испытаний также соответствует оператор $\widehat{\mathscr{H}}_{M}$, действующий на векторы (13) согласно соотношениям

$$
\widehat{\mathscr{H}}_{M} \Psi=\sum_{i_{1}=1}^{l} \cdots \sum_{i_{M}=1}^{l}\left(\sum_{s=1}^{M} \lambda_{i_{s}}\right) \psi\left(i_{1}, \ldots, i_{M}\right) \mathbf{e}_{i_{1}} \otimes \cdots \otimes \mathbf{e}_{i_{M}} .
$$

Введем для дальнейшего использования обозначения

$$
M_{i}\left(i_{1}, \ldots, i_{M}\right)=\sum_{s=1}^{M} \delta_{i i_{s}}
$$

где $\delta_{i i^{\prime}}$ - символ Кронекера. Теперь определим проекторы $\widehat{P}_{\{M\}}$ следующим образом:

$$
\widehat{P}_{\{M\}} \Psi=\sum_{i_{1}=1}^{l} \cdots \sum_{i_{M}=1}^{l}\left(\prod_{i=1}^{l} \delta_{M_{i} M_{i}\left(i_{1}, \ldots, i_{M}\right)}\right) \psi\left(i_{1}, \ldots, i_{M}\right) \mathbf{e}_{i_{1}} \otimes \cdots \otimes \mathbf{e}_{i_{M}},
$$

здесь $\{M\}$ обозначает произвольный набор неотрицательных целых чисел $M_{1}, \ldots, M_{l}$, удовлетворяющих условию

$$
\sum_{i=1}^{l} M_{i}=M
$$

Легко убедиться, что для операторов (17) справедливы равенства

$$
\widehat{P}_{\{M\}} \widehat{P}_{\left\{M^{\prime}\right\}}=\prod_{i=1}^{l} \delta_{M_{i} M_{i}^{\prime}} \widehat{P}_{\{M\}}
$$

и, таким образом, эти операторы являются проекторами. Оператор $\widehat{\mathscr{H}}_{M}(15)$ выражается через проекторы $\widehat{P}_{\{M\}}$ следуюшим образом:

$$
\widehat{\mathscr{H}}_{M}=\sum_{\{M\}}\left(\sum_{i=1}^{l} \lambda_{i} M_{i}\right) \widehat{P}_{\{M\}} .
$$


В подпространстве $\mathscr{L}_{\{M\}}$, на которое проектор $\widehat{P}_{\{M\}}$ проектирует пространство $\mathscr{L}_{M}$, существует выделенный базис, образованный векторами, имеющими вид произведения

$$
\mathrm{E}_{i_{1}, \ldots, i_{M}}=\mathbf{e}_{i_{1}} \otimes \cdots \otimes \mathbf{e}_{i_{M}}
$$

где $\mathbf{e}_{i}$ встречается $M_{i}$ раз, или, другими словами, набор $i_{1}, \ldots, i_{M}$ удовлетворяет условиям

$$
M_{i}\left(i_{1}, \ldots, i_{M}\right)=M_{i} .
$$

Введем следующую систему векторов $\Phi_{\{M\}}$ :

$$
\Phi_{\{M\}}=\widehat{P}_{\{M\}} \mathbf{e} \otimes \cdots \otimes \mathbf{e}, \quad \mathbf{e}=\sum_{i=1}^{l} \mathbf{e}_{i} .
$$

Векторы (23) равны сумме всех векторов (21) из подпространства $\mathscr{L}_{\{M\}}$, кроме того, имеет место равенство

$$
\Phi_{\{M\}}=\frac{1}{M !}\left(\prod_{i=1}^{l} M_{i} !\right) \operatorname{Symm}_{i_{1}, \ldots, i_{M}} \mathrm{E}_{i_{1}, \ldots, i_{M}}
$$

где $\mathrm{Symm}_{i_{1}, \ldots, i_{M}}$ - оператор симметризации по индексам $i_{1}, \ldots, i_{M}$.

Теперь определим понятия эволючии и сокращения информачии.

О п р е д е л е н и е 1 . Эволюиией ансамбля, состоящего из $M$ элементов - компонент вектора $\Psi \in \mathscr{L}_{M}$, за один шаг будем называть следующий вектор пространства $\mathscr{L}_{M}$ :

$$
\Psi_{1}=\exp \left(-\beta \widehat{\mathscr{H}}_{M}\right) \Psi
$$

где $\beta$ - некоторый параметр, означающий покупательную способность рубля (а значит, при $\beta \rightarrow \infty \Psi_{1}$ должно стремиться к нулю).

О п р е д е л е н и е 2. Сокращением информачии, или факторизацией, будем называть операцию, которая любому вектору $\Psi \in \mathscr{L}_{M}$ сопоставляет другой вектор по следующему закону:

$$
R(\Psi)=\sum_{\{M\}}\left\|\widehat{P}_{\{M\}} \Psi\right\| \Phi_{\{M\}},
$$

где суммирование ведется по всем наборам неотрицательных целых чисел $M_{1}, \ldots, M_{n}$, удовлетворяющих условию (18).

Каждому набору неотрицательных чисел $g_{i}, i=1, \ldots, l$, сопоставим следующий вектор пространства $\mathscr{L}_{M}$ :

$$
\Psi_{g}=\mathbf{g} \otimes \cdots \otimes \mathbf{g}
$$

где $\mathbf{g}$ - вектор пространства $L$, имеющий вид

$$
\mathrm{g}=\sum_{i=1}^{l} g_{i} \mathbf{e}_{i} .
$$


Для любого набора неотрицательных чисел $g_{i}, i=1, \ldots, l$, определим эволюцию векторов ансамбля из $M$ элементов $\Psi_{g}(n) \in \mathscr{L}_{\{M\}}$ следующими рекуррентными формулами:

$$
\Psi_{g}(n+1)=R\left(\exp \left(-\beta \widehat{\mathscr{H}}_{M}\right) \Psi_{g}(n)\right), \quad n=0,1,2, \ldots, \quad \Psi_{g}(0)=\Psi_{g} .
$$

Обозначим через $\psi_{g}\left(i_{1}, \ldots, i_{M} ; n\right)$ коэффициенты разложения (13) вектора $\Psi_{g}(n)$ по базису $(21)$. Иными словами, $\psi_{g}\left(i_{1}, \ldots, i_{M} ; n\right)$ и $\Psi_{g}(n)$ связаны равенством

$$
\Psi_{g}(n)=\sum_{i_{1}=1}^{l} \cdots \sum_{i_{M}=1}^{l} \psi_{g}\left(i_{1}, \ldots, i_{M} ; n\right) \mathbf{e}_{i_{1}} \otimes \cdots \otimes \mathbf{e}_{i_{M}} .
$$

Функцию $\psi_{g}\left(i_{1}, \ldots, i_{M} ; n\right)$ будем считать ненормированной функцией распределения факторизованного ансамбля через $n$ шагов при условии, что в начальный момент функция распределения факторизованного ансамбля имела вид произведения: $\psi_{g}\left(i_{1}, \ldots, i_{M} ; 0\right)=g_{i_{1}} \cdots g_{i_{M}}$. В таком случае $g_{i}$ имеет смысл ненормированной функции распределения для одной системы факторизованного ансамбля. Рассмотрим следующие функции:

$$
\begin{aligned}
F(n, g, M) & =-\frac{1}{M \beta(n+1)} \ln \left(\left\|\Psi_{g}(n)\right\|\right), \\
w_{i}(n, g, M) & =\frac{1}{\left\|\Psi_{g}(n)\right\|} \sum_{i_{2}=1}^{l} \cdots \sum_{i_{M}=1}^{l} \psi_{g}\left(i, i_{2}, \ldots, i_{M} ; n\right) .
\end{aligned}
$$

Так как $\psi_{g}\left(i_{1}, \ldots, i_{M}\right)$ является функцией распределения для факторизованного ансамбля из $M$ систем, то формула (32) выражает функцию распределения одной системы. Рассмотрим пределы функций распределения (31) и (32) для одной системы факторизованного ансамбля, когда число его элементов $M$ стремится к бесконечности.

Теорема 1. При $M \rightarrow \infty$ функиии распределения $F(n, g, M)$ $u w_{i}(n, g, M)$ имеют следуюиие предель:

$$
\begin{aligned}
& \lim _{M \rightarrow \infty} F(n, g, M)=-\frac{1}{\beta} \ln \left(\sum_{i=1}^{l} e^{-n \beta \lambda_{i} /(n+1)} g_{i}^{1 /(n+1)}\right) \equiv \widetilde{F}(n, g), \\
& \lim _{M \rightarrow \infty} w_{i}(n, g, M)=\frac{e^{-n \beta \lambda_{i} /(n+1)} g_{i}^{1 /(n+1)}}{\sum_{j=1}^{l} e^{-n \beta \lambda_{j} /(n+1)} g_{j}^{1 /(n+1)}} \equiv \widetilde{w}_{i}(n, g) .
\end{aligned}
$$

Д о к а з а т е л ь с т в о. Вектор (27) представляется в виде

$$
\Psi_{g}=\sum_{\{M\}}\left(\prod_{i=1}^{l} g_{i}^{M_{i}}\right) \Phi_{\{M\}}
$$


Подставив (35) в (29), получим

$$
\Psi_{g}(n)=(M !)^{l} \sum_{\{M\}}\left(\prod_{i=1}^{l} \frac{g_{i}^{M_{i}} e^{-n \beta \lambda_{i} M_{i}}}{\left(M_{i} !\right)^{n}}\right) \Phi_{\{M\}} .
$$

Из (36) следует

$$
\psi_{g}\left(i_{1}, \ldots, i_{M} ; n\right)=(M !)^{l}\left(\prod_{i=1}^{l} \frac{\left(g_{i} e^{-n \beta \lambda_{i}}\right)^{M_{i}}}{\left(M_{i} !\right)^{n}}\right),
$$

где $M_{i}$ выражается через $i_{1}, \ldots, i_{M}$ согласно (16). Подставляя выражения (37) в (14), а также суммируя (37) по всем индексам кроме одного, получим

$$
\begin{aligned}
& \left\|\Psi_{g}(n)\right\|=(M !)^{n+1} \sum_{\{M\}} \prod_{i=1}^{l} \frac{g_{i}^{M_{i}} e^{-n \beta \lambda_{i} M_{i}}}{\left(M_{i} !\right)^{n+1}} \\
& \sum_{i_{2}=1}^{l} \cdots \sum_{i_{M}=1}^{l} \psi_{g}\left(i, i_{2}, \ldots, i_{M} ; n\right) \\
& =(M !)^{n+1} \sum_{\{M\}}\left(\prod_{i=1}^{l} \frac{g_{i}^{M_{i}} e^{-n \beta \lambda_{i} M_{i}}}{\left(M_{i} !\right)^{n+1}}\right)\left(\sum_{j=1}^{l} \frac{M_{j}}{M}\right) .
\end{aligned}
$$

Для нахождения асимптотики при $M \rightarrow \infty$ сумм в формулах (38), (39) используем метод Лапласа [8], [9]. Применение этого метода дает следующие равенства:

$$
\begin{aligned}
\lim _{M \rightarrow \infty} & \frac{1}{M} \ln \left(\left\|\Psi_{p, \lambda}(n)\right\|\right)=(n+1) \ln \left(\sum_{i=1}^{l} e^{-n \beta \lambda_{i} /(n+1)} g_{i}^{1 /(n+1)}\right), \\
\lim _{M \rightarrow \infty} & \frac{1}{\left\|\Psi_{p, \lambda}(n)\right\|} \sum_{i_{2}=1}^{l} \cdots \sum_{i_{M}=1}^{l} \psi_{p, \lambda}\left(i, i_{2}, \ldots, i_{M} ; n\right) \\
= & \frac{e^{-n \beta \lambda_{i} /(n+1)} g_{i}^{1 /(n+1)}}{\sum_{j=1}^{l} e^{-n \beta \lambda_{j} /(n+1)} g_{j}^{1 /(n+1)}} .
\end{aligned}
$$

Подстановка равенств (40), (41) в формулы (31), (32) приводит к (33), (34). Теорема доказана.

Таким образом, в силу (34) нормированное распределение $\widetilde{w}_{i}(0, g)=$ $g_{i} / \sum_{j=1}^{l} g_{j}$ за $n$ шагов эволюции переходит в $\widetilde{w}_{i}(n, g)$.

Рассмотрим теперь выражения $\tilde{F}(n, g)(33)$ и $\widetilde{w}_{i}(n, g)$ (34) при $n \rightarrow \infty$, т.е. предельные распределения. Пусть $I$ - непустое подмножество множества $1, \ldots, l$, и пусть для $g_{i}$ выполняются условия

$$
g_{i}>0 \quad \text { при } \quad i \in I, \quad g_{i}=0 \text { при } i \notin I .
$$


В таком случае из (33), (34) следует

$$
\begin{aligned}
& \lim _{n \rightarrow \infty} \tilde{F}(n, g)=-\frac{1}{\beta} \ln \left(\sum_{i \in I} e^{-\beta \lambda_{i}}\right), \\
& \lim _{n \rightarrow \infty} \widetilde{w}_{i}(n, g)=\frac{e^{-\beta \lambda_{i}}}{\sum_{j=1}^{l} e^{-\beta \lambda_{j}}} \equiv \rho_{i} .
\end{aligned}
$$

В случае, когда множество $I$ совпадает с множеством $\{1, \ldots, l\}$, выражения (43) и (44) являются соответственно свободной энергией и распределением Гиббса при температуре $1 / \beta$ для системы, уровни энергии которой невырождены и равны $\lambda_{1}, \ldots, \lambda_{l}$. В случае, когда $I$ меньше, чем множество $\{1, \ldots, l\}$, выражения (43) и (44) соответствуют распределению системы не по всем уровням, а по части. Заметим также, что если для $g_{i}$ выполняется

$$
g_{i}=\left\{\begin{array}{lll}
A e^{-\beta \lambda_{i}} & \text { при } & i \in I, \\
0 & \text { при } & i \notin I,
\end{array}\right.
$$

где $A$ - произвольное положительное число, то $\widetilde{w}_{i}(n, g)(34)$ совпадает с $\rho_{i}$ (44) при любом $n$. Таким образом, полное и частичные распределения Гиббса оказываются инвариантными относительно приведенной эволюции. В случае $\beta<0$ формула (43) совпадает с финансовым осреднением дохода [5], [10], [11] (с точностью до константы), при $\beta>0-\mathrm{c}$ финансовым осреднением расхода.

Рассмотрим частный случай, встречающийся в финансовых задачах, когда случайная величина $\lambda$ принимает значение полинома:

$$
\mathscr{E}\left(N_{1}\right)=\sum_{q=0}^{p} A_{q} N_{1}^{q}
$$

где $N_{1}$ принимает значения $0,1, \ldots, N$. Исследуем вопрос о выполнимости условия (12) в этом случае.

Утверждение 1. B случае, когда $p<N$, условие (12) не выполняется ни при каких значениях коэффиииентов $A_{q}$, а в случае, когда $p \geqslant N$, условие (12) выполняется для коэффичиентов $A_{q}$ в общем положении.

Д о к а з а т е л ь с в о. В рассматриваемом случае условие (12) принимает следующий вид: не существует ненулевого набора целых чисел $P_{0}, P_{1}, \ldots, P_{N}$, для которого одновременно выполняются равенства

$$
\sum_{N_{1}=0}^{N} \mathscr{E}\left(N_{1}\right) P_{N_{1}}=0, \quad \sum_{N_{1}=0}^{N} P_{N_{1}}=0 .
$$

По-другому это условие можно сформулировать так: не существует набора целых чисел $P_{1}, \ldots, P_{N}$, для которого выполняется равенство

$$
\sum_{N_{1}=1}^{N}\left(\mathscr{E}\left(N_{1}\right)-\mathscr{E}(0)\right) P_{N_{1}}=\sum_{q=1}^{p} A_{q}\left(\sum_{N_{1}=1}^{N} N_{1}^{q} P_{N_{1}}\right)=0
$$


В курсе линейной алгебры доказано, что определитель матрицы Вандермонда

$$
\left(\begin{array}{cccc}
1 & 1 & \cdots & 1 \\
x_{1} & x_{2} & \cdots & x_{N} \\
x_{1}^{2} & x_{2}^{2} & \cdots & x_{N}^{2} \\
\vdots & \vdots & \ddots & \vdots \\
x_{1}^{N-1} & x_{2}^{N-1} & \cdots & x_{N}^{N-1}
\end{array}\right)
$$

отличен от нуля, если $x_{i} \neq x_{j}$ при $i \neq j$, а кроме того, если числа $x_{i}$ целые, то обратная к (49) матрица состоит из рациональных чисел. Отсюда следует, что при $p<N$ система уравнений

$$
\sum_{N_{1}=1}^{N} N_{1}^{q} P_{N_{1}}=0, \quad q=1, \ldots, p,
$$

имеет ненулевые целые решения и, следовательно, существует ненулевой набор целых чисел $P_{1}, \ldots, P_{N}$, для которого выполняется равенство (48). Таким образом, первая часть утверждения доказана. В случае, когда $p \geqslant N$, из указанных свойств матрицы (49) следует, что не существует ненулевого решения системы уравнений (50). Поэтому для любого ненулевого набора цельх чисел $P_{1}, \ldots, P_{N}$ хотя бы одно из чисел

$$
J_{q}(P)=\sum_{N_{1}=1}^{N} N_{1}^{q} P_{N_{1}}, \quad q=1, \ldots, p,
$$

отлично от нуля. Отсюда следует, что если при $p \geqslant N$ существует ненулевой набор целых чисел $P_{1}, \ldots, P_{N}$, для которого выполняется равенство (48), то коэффициенты $A_{q}$ не находятся в общем положении. А именно, в этом случае существуют число $1 \leqslant q_{0} \leqslant p$ и набор рациональных чисел $R_{q}, q \neq q_{0}$, такие, что

$$
A_{q_{0}}=\sum_{1 \leqslant q \leqslant N, q \neq q_{0}} A_{q} R_{q}
$$

При фиксированных коэффициентах $A_{q}, q \neq q_{0}$, в силу рациональности чисел $R_{q}, q \neq q_{0}$, правая часть равенства (52) принимает только счетное число значений, а в общем положении коэффициент $A_{q_{0}}$ может принимать несчетное множество значений. Таким образом, равенство (48) может быть выполнено только не в общем положении.

3. Зависимость покупательной способности и среднего дохода населения от числа покупателей на специализированном рынке и в регионе. Распределение Парето. Рассмотрим рынок каких-либо специализированных товаров, например, авторынок. На рынке имеется $n$ различных товаров, цена товара с номером $j$ равна $\lambda_{j}$ рублей. Пусть имеется $N$ покупателей, которые покупают какой-либо 
один предмет. Обозначим через $N_{j}, j=1, \ldots, n$, число покупателей, купивших товар с номером $j$; таким образом,

$$
\sum_{j=1}^{n} N_{j}=N
$$

Доход, полученный продавцом, выражается формулой $\mathscr{E}(\{N\})=$ $\sum_{j=1}^{n} \lambda_{j} N_{j}$, где $\{N\}$ обозначает набор целых неотрицательных чисел $N_{1}, \ldots, N_{n}$, удовлетворяющих условию (53).

Глобальное финансовое осреднение имеет вид (см. [6]) $\mathbb{E}=\beta^{-1} \times$ $\ln \left(\sum_{\{N\}} \alpha(\{N\}) \exp (\beta \mathscr{E}(\{N\}))\right)$, где $\alpha(\{N\})$ - зависящие от набора $\{N\}$ неотрицательные числа, которые удовлетворяют условию нормировки: $\sum_{\{N\}} \alpha(\{N\})=1, \beta>0$ - покупательная способность рубля, кроме того, $\sum_{\{N\}}$ обозначает сумму по всем наборам $\{N\}$, удовлетворяющим условию (87) (см. ниже п. 4).

В нашем случае перестановка покупателей сказывается на числе вариантов. В физике это значит, что они подчиняются больцмановской статистике и, как следует из аксиоматики, в этом случае веса $\alpha(\{N\})$ имеют вид $\alpha(\{N\})=C \prod_{j=1}^{n} 1 / N_{j}$ !, где число $C$ определяется из условия нормировки. Используем здесь и далее следуюшее хорошо известное равенство:

$$
\sum_{\{N\}}\left(\prod_{j=1}^{n} \frac{a_{j}^{N_{j}}}{N_{j} !}\right)=\frac{1}{N !}\left(\sum_{j=1}^{n} a_{j}\right)^{N}
$$

которое справедливо для произвольных чисел $a_{j}, j=1, \ldots, n$. Из (54) следует, что $C=N ! / n^{N}$.

Следовательно, глобальное финансовое осреднение имеет вид

$$
\mathbb{E}=\frac{1}{\beta} \ln \left(\frac{N !}{n^{N}} \sum_{\{N\}} \exp \left(\beta \sum_{j=1}^{n} \lambda_{j} N_{j}\right)\left(\prod_{k=1}^{n} \frac{1}{N_{k} !}\right)\right) .
$$

Используя (54), приведем окончательно глобальное финансовое осреднение (86) к виду

$$
\mathbb{E}=\frac{N}{\beta} \ln \left(\frac{1}{n} \sum_{j=1}^{n} e^{\beta \lambda_{j}}\right)
$$

Исследуем асимптотику глобального финансового осреднения при $N \rightarrow \infty$. Без ограничения общности будем дальше считать, что $\lambda_{j}=\lambda(j / n), j=1, \ldots, n$, где функция $\lambda(x) \in C^{\infty}([0,1])$ не зависит от $N$. Кроме того, предположим, что $n=N^{\alpha}$, где $\alpha>0$. Для того чтобы найти асимптотику выражения (56), найдем сначала асимптотику при $n \rightarrow \infty$ суммы $n^{-1} \sum_{j=1}^{n} e^{\beta \lambda_{j}}$. 
Очевидно, что для произвольной функции $g(x) \in C^{\infty}([0,1])$ выполняется равенство

$$
\frac{1}{n} \sum_{j=1}^{n} g\left(\frac{j}{n}\right)=\int_{0}^{1} g(x) d x+\frac{g(1)-g(0)}{2 n}+2 \sum_{k=1}^{\infty} \int_{0}^{1} g(x) \cos (2 \pi k n x) d x
$$

(cм. [8]).

Кроме того, для любых целых $k, n \neq 0$ имеем

$$
\int_{0}^{1} g(x) \cos (2 \pi k n x) d x=\frac{g^{\prime}(1)-g^{\prime}(0)}{(2 \pi k n)^{2}}-\frac{1}{(2 \pi k n)^{3}} \int_{0}^{1} g^{\prime \prime \prime}(x) \sin (2 \pi k n x) d x .
$$

Подставим (58) в (57) и учтем, что $\sum_{k=1}^{\infty} 1 / k^{2}=\pi^{2} / 6$, в результате получим

$$
\begin{aligned}
\frac{1}{n} \sum_{j=1}^{n} g\left(\frac{j}{n}\right)= & \int_{0}^{1} g(x) d x+\frac{g(1)-g(0)}{2 n}+\frac{g^{\prime}(1)-g^{\prime}(0)}{12 n^{2}} \\
& +\frac{2}{(2 \pi n)^{3}} \sum_{k=1}^{\infty} \frac{1}{k^{3}} \int_{0}^{1} g^{\prime \prime \prime}(x) \sin (2 \pi k n x) d x
\end{aligned}
$$

Заметим теперь, что для любых $k$ и $g(x) \in C^{\infty}([0,1])$ при $n \rightarrow \infty$ справедливо

$$
\int_{0}^{1} g^{\prime \prime \prime}(x) \sin (2 \pi k n x) d x=o(1)
$$

Учитывая (59) и (60), а также то, что $\lambda_{j}=\lambda(j / n)$, из тождеств $(8.38)$, (8.56) книги [8] получаем, что

$$
\begin{aligned}
\frac{1}{n} \sum_{j=1}^{n} e^{\beta \lambda_{j}}= & \int_{0}^{1} e^{\beta \lambda(x)} d x+\frac{1}{2 n}\left(e^{\beta \lambda(1)}-e^{\beta \lambda(0)}\right) \\
& +\frac{\beta}{12 n^{2}}\left(e^{\beta \lambda(1)} \lambda^{\prime}(1)-e^{\beta \lambda(0)} \lambda^{\prime}(0)\right)+O\left(\frac{1}{n^{3}}\right) .
\end{aligned}
$$

Подставим (61) в (56). Поскольку $\ln (1+x)=x-x^{2} / 2+x^{3} / 3+O\left(x^{4}\right)$, a $n=N^{\alpha}$, то асимптотика глобального финансового осреднения при $N \rightarrow \infty$ имеет вид

$$
\begin{aligned}
\mathbb{E}= & \frac{N}{\beta} \ln \left(\int_{0}^{1} e^{\beta \lambda(x)} d x\right)+\frac{1}{2 \beta N^{\alpha-1}} \frac{e^{\beta \lambda(1)}-e^{\beta \lambda(0)}}{\int_{0}^{1} e^{\beta \lambda(x)} d x} \\
& +\frac{1}{12 N^{2 \alpha-1}} \frac{e^{\beta \lambda(1)} \lambda^{\prime}(1)-e^{\beta \lambda(0)} \lambda^{\prime}(0)}{\int_{0}^{1} e^{\beta \lambda(x)} d x}-\frac{1}{8 \beta N^{2 \alpha-1}}\left(\frac{e^{\beta \lambda(1)}-e^{\beta \lambda(0)}}{\int_{0}^{1} e^{\beta \lambda(x)} d x}\right)^{2} \\
& +O\left(\frac{1}{N^{3 \alpha-1}}\right) .
\end{aligned}
$$

Получим значение величины $m$, сопряженной к числу покупателей $N$. Эту величину будем называть средним покупательным потенииалом. Согласно приведенной в [6] концепции, для этого продифференцируем глобальное финансовое осреднение по $N$. Из (62) получим, что в 
случае $\alpha \neq 1$ первые два члена асимптотики $m$ при $N \rightarrow \infty$ имеют вид:

$$
m=\frac{1}{\beta} \ln \left(\int_{0}^{1} e^{\beta \lambda(x)} d x\right)+\frac{(1-\alpha)}{2 \beta N^{\alpha}} \frac{e^{\beta \lambda(1)}-e^{\beta \lambda(0)}}{\int_{0}^{1} e^{\beta \lambda(x)} d x}+O\left(\frac{1}{N^{2 \alpha}}\right) .
$$

Если $\alpha=1$, то из (62) следует, что

$$
\begin{aligned}
m= & \frac{1}{\beta} \ln \left(\int_{0}^{1} e^{\beta \lambda(x)} d x\right)-\frac{1}{12 N^{2}} \frac{e^{\beta \lambda(1)} \lambda^{\prime}(1)-e^{\beta \lambda(0)} \lambda^{\prime}(0)}{\int_{0}^{1} e^{\beta \lambda(x)} d x} \\
& +\frac{1}{8 \beta N^{2}}\left(\frac{e^{\beta \lambda(1)}-e^{\beta \lambda(0)}}{\int_{0}^{1} e^{\beta \lambda(x)} d x}\right)^{2}+O\left(\frac{1}{N^{3}}\right) .
\end{aligned}
$$

Мы получаем распределение Парето, что согласуется со статистическими данными [12]-[14].

Будем далее считать, что количество каждого товара в магазине ограничено и равно $s \geqslant N / n$. Такому случаю соответствует следующее локальное финансовое осреднение:

$$
\mathbb{E}(s)=\frac{1}{\beta} \ln \left(\frac{1}{C(s)} \sum_{\{N\}, s} \exp \left(\beta \sum_{j=1}^{n} \lambda_{j} N_{j}\right)\left(\prod_{k=1}^{n} \frac{1}{N_{k} !}\right)\right),
$$

где $\sum_{\{N\}, s}$ обозначает сумму по всем наборам целых чисел $0 \leqslant N_{j} \leqslant s$, $j=1, \ldots, n$, удовлетворяющим условию (53), а число $C(s)$, согласно условию нормировки, имеет вид

$$
C(s)=\sum_{\{N\}, s}\left(\prod_{j=1}^{n} \frac{1}{N_{j} !}\right)
$$

Утверждение 2. Для сумм в формуле (65) справедливо тождество

$$
\begin{aligned}
\Sigma(s) & \equiv \sum_{\{N\}, s} \exp \left(\beta \sum_{j=1}^{n} \lambda_{j} N_{j}\right)\left(\prod_{k=1}^{n} \frac{1}{N_{k} !}\right) \\
& =\frac{A^{N}}{2 \pi} \int_{-\pi}^{\pi} d \alpha e^{-i N \alpha} \exp \left(\sum_{j=1}^{n} \varphi_{s}\left(e^{\beta \lambda_{j}} e^{i \alpha} / A\right)\right),
\end{aligned}
$$

где $A \neq 0$ - произвольное число, а функиия $\varphi_{s}$ имеет вид $\varphi_{s}(y)=$ $\ln \left(\sum_{n=0}^{s} y^{n} / n !\right)$.

Д ок а з а т е л с с т в о. Заметим, что для $\Sigma(s)$ выполняются следующие равенства:

$$
\begin{aligned}
\Sigma(s) & =\sum_{N_{1}=0}^{s} \cdots \sum_{N_{n}=0}^{s} \Delta\left(N-\sum_{j=1}^{n} N_{j}\right)\left(\prod_{k=1}^{n} \frac{e^{\beta \lambda_{k} N_{k}}}{N_{k} !}\right) \\
& =A^{N} \sum_{N_{1}=0}^{s} \cdots \sum_{N_{n}=0}^{s} \Delta\left(\sum_{j=1}^{n} N_{j}-N\right)\left(\prod_{k=1}^{n} \frac{e^{\beta \lambda_{k} N_{k}}}{A^{N_{k}} N_{k} !}\right),
\end{aligned}
$$


где $A \neq 0$ - произвольное число, а $\Delta(k)$ - функция целочисленного аргумента, равная 1 при $k=0$ и 0 при $k \neq 0$ :

$$
\Delta(k)=\frac{1}{2 \pi} \int_{-\pi}^{\pi} d \alpha e^{i k \alpha} .
$$

Отсюда

$$
\Sigma(s)=\frac{A^{N}}{2 \pi} \int_{-\pi}^{\pi} d \alpha e^{-i N \alpha} \prod_{j=1}^{n}\left(\sum_{N_{j}=0}^{s} \frac{1}{N_{j} !}\left(\frac{e^{\beta \lambda_{j}} e^{i \alpha}}{A}\right)^{N_{j}}\right) .
$$

Таким образом, равенство (70) совпадает с равенством (67).

Заметим, что $\Sigma(s)$ (см. 67) при $\beta=0$ совпадает с $C(s)$ (см. 66). Поэтому $C(s)$ в силу доказанного выше утверждения представляется в виде

$$
C(s)=\frac{B^{N}}{2 \pi} \int_{-\pi}^{\pi} d \alpha \exp \left(-i N \alpha+n \varphi_{s}\left(\frac{e^{i \alpha}}{B}\right)\right) .
$$

Введенная выше функция $\varphi_{s}(x)$ бесконечно дифференцируема при $x>0$ и обладает следующими свойствами: $\left(x \varphi_{s}(x)\right)^{\prime}=$ $x \varphi_{s}^{\prime \prime}(x)+\varphi_{s}^{\prime}(x)>0$ при $x>0, \lim _{x \rightarrow+0}\left(x \varphi_{s}^{\prime}(x)\right)=0, \lim _{x \rightarrow+\infty}\left(x \varphi_{s}^{\prime}(x)\right)=s$.

Найдем асимптотику локального финансового осреднения при $N \rightarrow \infty$ в случае $n=N, s>1$. В этом случае интегралы в формулах (67) и (71) содержат экспоненту от величины порядка $N$, поэтому асимптотика локального финансового осреднения при $N \rightarrow \infty$ может быть найдена с помощью метода, развитого в теории комплексного ростка [8].

Рассмотрим сначала асимптотику $C(s)$. Заметим, что при $n=N$ интеграл в формуле (71) представляется в виде $\int_{-\pi}^{\pi} d \alpha e^{N S(\alpha, B)}$, где $S(\alpha, B)=-i \alpha+\varphi_{s}\left(e^{i \alpha} / B\right)$. Поскольку число $B$ в равенстве $(71)$ является произвольным, то положим, что $B=B_{s}$, где $B_{s}$ - положительное решение следующего уравнения:

$$
\varphi_{s}^{\prime}\left(\frac{1}{B_{s}}\right) \frac{1}{B_{s}}=1 .
$$

Уравнение (72) при $s>1$ имеет положительное решение, а такое решение единственно. Из (72) следует, что $S^{\prime}\left(0, B_{s}\right)=0$, если $B=B_{s}$, кроме того, непосредственными вычислениями легко убедиться, что $S^{\prime \prime}\left(0, B_{s}\right)$ - действительное отрицательное число, а функция $\operatorname{Re} S\left(\alpha, B_{s}\right)$ имеет максимум по переменной $\alpha$ при $\alpha=0$. Здесь и далее во всех формулах все производные функции $S(\alpha, B)$ берутся по переменной $\alpha$. Сформулированные свойства функции $S(\alpha, B)$ позволяют найти асимптотику интеграла:

$$
\begin{aligned}
\int_{-\pi}^{\pi} d \alpha e^{N S\left(\alpha, B_{s}\right)}= & e^{N S\left(0, B_{s}\right)} \sqrt{\frac{2 \pi}{-N S^{\prime \prime}\left(0, B_{s}\right)}} \\
& \times\left(1+\frac{S^{\prime \prime \prime \prime}\left(0, B_{s}\right)}{8 N\left(S^{\prime \prime}\left(0, B_{s}\right)\right)^{2}}-\frac{5\left(S^{\prime \prime \prime}\left(0, B_{s}\right)\right)^{2}}{24 N\left(S^{\prime \prime}\left(0, B_{s}\right)\right)^{3}}+O\left(\frac{1}{N^{2}}\right)\right) .
\end{aligned}
$$


Умножая, согласно равенству (71), левую часть равенства (73) на $B_{s}^{N} /(2 \pi)$, получим асимптотику $C(s)$ при $N \rightarrow \infty$.

Теперь найдем, исходя из тех же формул (8.38) и (8.56) книги [8], асимптотику $\Sigma(s)$. Для этого рассмотрим интеграл

$$
\int_{-\pi}^{\pi} d \alpha e^{-i N \alpha} \exp \left(\sum_{j=1}^{n} \varphi_{s}\left(\frac{e^{\beta \lambda_{j}} e^{i \alpha}}{A}\right)\right) .
$$

Из (61) следует, что интеграл (74) представляется в виде

$$
\int_{-\pi}^{\pi} d \alpha \exp \left(N S_{0}(\alpha, A)+S_{1}(\alpha, A)+\frac{1}{N} S_{2}(\alpha, A)+\frac{1}{N^{2}} S_{3}(\alpha, A)\right),
$$

где функции $S_{0}(\alpha, A), S_{1}(\alpha, A), S_{2}(\alpha, A), S_{3}(\alpha, A)$ выражаются следующим образом:

$$
\begin{aligned}
S_{0}(\alpha, A)= & -i \alpha+\int_{0}^{1} \varphi_{s}\left(\frac{e^{\beta \lambda(x)} e^{i \alpha}}{A}\right) d x \\
S_{1}(\alpha, A)= & \frac{1}{2}\left(\varphi_{s}\left(\frac{e^{\beta \lambda(1)} e^{i \alpha}}{A}\right)-\varphi_{s}\left(\frac{e^{\beta \lambda(0)} e^{i \alpha}}{A}\right)\right), \\
S_{2}(\alpha, A)= & \frac{\beta e^{i \alpha}}{12 A}\left(\varphi_{s}^{\prime}\left(\frac{e^{\beta \lambda(1)} e^{i \alpha}}{A}\right) e^{\beta \lambda(1)} \lambda^{\prime}(1)\right. \\
& \left.-\varphi_{s}\left(\frac{e^{\beta \lambda(0)} e^{i \alpha}}{A}\right) e^{\beta \lambda(0)} \lambda^{\prime}(0)\right), \\
S_{3}(\alpha, A)= & O(1) .
\end{aligned}
$$

Здесь число $A$ произвольное. В частности, его можно положить для удобства равным $A_{s}$, где $A_{s}$ - положительное решение уравнения

$$
\int_{0}^{1} \varphi_{s}^{\prime}\left(\frac{e^{\beta \lambda(x)}}{A_{s}}\right) \frac{e^{\beta \lambda(x)}}{A_{s}} d x=1 .
$$

Только в этом случае мы сможем воспользоваться асимптотическими формулами, используюшими теорию комплексного ростка [8]. Здесь $\varphi_{s}^{\prime}$ - производная по аргументу, стоящему в скобках.

Уравнение (80) имеет единственное положительное решение. Непосредственные вычисления позволяют убедиться, что $S_{0}^{\prime}\left(\alpha, A_{s}\right)=0$, $S_{0}^{\prime \prime}\left(\alpha, A_{s}\right)$ - действительное отрицательное число и функция $\operatorname{Re} S_{0}\left(\alpha, A_{s}\right)$ имеет максимум по переменной $\alpha$ в точке $\alpha=0$. Отметим, что здесь и далее во всех формулах производные функций $S_{l}(\alpha, A), l=1,2,3$, берутся по переменной $\alpha$. Свойства функции $S_{0}\left(\alpha, A_{s}\right)$ позволяют найти следуюшую асимптотику интеграла (74), (75) при $N \rightarrow \infty$ :

$$
\int_{-\pi}^{\pi} d \alpha e^{-i N \alpha} \exp \left(\sum_{j=1}^{n} \varphi_{s}\left(\frac{e^{\beta \lambda_{j}} e^{i \alpha}}{A}\right)\right)=e^{N S_{0}\left(0, A_{s}\right)} e^{S_{1}\left(0, A_{s}\right)} \sqrt{\frac{2 \pi}{-N S_{0}^{\prime \prime}\left(0, A_{s}\right)}}
$$




$$
\begin{aligned}
\times(1 & +\frac{S_{2}\left(0, A_{s}\right)}{N}+\frac{S_{0}^{\prime \prime \prime}\left(0, A_{s}\right)}{8 N\left(S_{0}^{\prime \prime}\left(0, A_{s}\right)\right)^{2}}-\frac{5\left(S_{0}^{\prime \prime \prime}\left(0, A_{s}\right)\right)^{2}}{24 N\left(S_{0}^{\prime \prime}\left(0, A_{s}\right)\right)^{3}} \\
& \left.-\frac{\left(S_{1}^{\prime}\left(0, A_{s}\right)\right)^{2}}{2 N S_{0}^{\prime \prime}\left(0, A_{s}\right)}+\frac{S_{0}^{\prime \prime \prime}\left(0, A_{s}\right) S_{1}^{\prime}\left(0, A_{s}\right)}{2 N\left(S_{0}^{\prime \prime}\left(0, A_{s}\right)\right)^{2}}+O\left(\frac{1}{N^{2}}\right)\right) .
\end{aligned}
$$

Чтобы получить асимптотику $\Sigma(s)$, в силу (67) достаточно умножить правую часть равенства $(81)$ на $A_{s}^{N} /(2 \pi)$.

Подстановка полученных асимптотик $C(s)$ и $\Sigma(s)$ в формулу (65) и разложение логарифма позволяют получить асимптотику локального финансового ожидания, аналогичную асимптотике (62) при $\alpha=1$ :

$$
\mathbb{E}(s)=N D_{0}(s)+D_{1}(s)+\frac{1}{N} D_{2}(s)+O\left(\frac{1}{N^{2}}\right),
$$

где коэффициенты $D_{l}(s), l=0,1,2$, выражаются следующим образом:

$$
\begin{aligned}
D_{0}(s)= & S_{0}\left(0, A_{s}\right)-S\left(0, B_{s}\right)+\ln \left(\frac{A_{0}}{B_{0}}\right) \\
D_{1}(s)= & S_{1}\left(0, A_{s}\right)+\ln \left(\frac{S^{\prime \prime}\left(0, B_{s}\right)}{S_{0}^{\prime \prime}\left(0, A_{s}\right)}\right) \\
D_{2}(s)= & \frac{S_{2}\left(0, A_{s}\right)}{N}+\frac{S_{0}^{\prime \prime \prime}\left(0, A_{s}\right)}{8 N\left(S_{0}^{\prime \prime}\left(0, A_{s}\right)\right)^{2}}-\frac{5\left(S_{0}^{\prime \prime \prime}\left(0, A_{s}\right)\right)^{2}}{24 N\left(S_{0}^{\prime \prime}\left(0, A_{s}\right)\right)^{3}} \\
& -\frac{\left(S_{1}^{\prime}\left(0, A_{s}\right)\right)^{2}}{2 N S_{0}^{\prime \prime}\left(0, A_{s}\right)}+\frac{S_{0}^{\prime \prime \prime}\left(0, A_{s}\right) S_{1}^{\prime}\left(0, A_{s}\right)}{2 N\left(S_{0}^{\prime \prime}\left(0, A_{s}\right)\right)^{2}}-\frac{S^{\prime \prime \prime \prime}\left(0, B_{s}\right)}{8 N\left(S^{\prime \prime}\left(0, B_{s}\right)\right)^{2}} \\
& +\frac{5\left(S^{\prime \prime \prime}\left(0, B_{s}\right)\right)^{2}}{24 N\left(S^{\prime \prime}\left(0, B_{s}\right)\right)^{3}}+O\left(\frac{1}{N^{2}}\right) .
\end{aligned}
$$

Дифференцирование асимптотики (82) по $N$ приводит к выражению, аналогичному (64). Таким образом, для локального среднего покупательного потенциала также имеет место распределение Парето.

Далее мы хотим определить по осредненному покупательному потенциалу на отдельных рынках специализированных товаров все средние расходы среднего покупателя по всем рынкам. и тем самым определить его средний доход. Ясно, что мы должны сложить все расходы (покупательные потенциалы) по всем рынкам. Действительно, расходы и покупательные способности гражданина относительно автомобиля и хлеба должны складываться, но по какому закону сложения? Очевидно, в силу построенной нелинейной арифметики, они должны складываться именно по тому же нелинейному закону сложения.

Такой способ сложения отличается от глобального математического осреднения только нормировкой. Поэтому общая сумма $\widetilde{\mathbb{E}}_{k}$ отличается от (55) только дополнительным слагаемым: $\widetilde{\mathbb{E}}_{k}=\mathbb{E}+N \ln (n / k)$. Если $k$ не зависит от $N$, а $n=N^{\alpha}, \alpha>0$, то второе слагаемое в формуле дает главный вклад в асимптотику при $N \rightarrow \infty$. Сопряженная к $N$ величина 
в этом случае принимает вид $\widetilde{m}_{k}=\alpha \ln (N)+m+\alpha(1-\ln (k))$, где $m$ выражается формулой (63) или (64). Выражение $\widetilde{m}_{k}$ логарифмически растет с ростом $N$, это означает, что средний доход населения данного региона логарифмически возрастает с ростом населения, что совпадает со статистическими данными [14].

4. Расходы покупателей и скорость оборота при нелинейном финансовом осреднении. Рассмотрим следуюшую модель. На специализированном рынке, или в специализированном магазине, имеется $n \geqslant 2$ различных товаров, цену товара с номером $j=1, \ldots, n$ обозначим $\lambda_{j}$, кроме того, будем считать, что $0<\lambda_{j}<\lambda_{j+1}$. Пусть товары разделены на две группы следуюшим образом: в первую группу входят товары с номерами $1 \leqslant j \leqslant n_{1}$, а во вторую группу - с номерами $n_{1}+1 \leqslant j \leqslant n$, здесь $1 \leqslant n_{1} \leqslant n-1-$ некоторое целое число. Будем считать, что на рынке имеется $S_{1}$ штук каждого товара из первой группы, а каждого товара из второй группы имеется $S_{2}$ штук. Пусть на рынок приходят $N \leqslant n_{1} S_{1}+n_{2} S_{2}$, где $n_{2}=n-n_{1}$, покупателей, каждый из которых покупает одну вещь. Рассмотрим финансовое осреднение расхода покупателей. Пусть товар с номером $j=1, \ldots, n$ купили $N_{j}$ человек, в таком случае общий расход покупателей равен

$$
\mathscr{E}(\{N\})=\sum_{j=1}^{n} \lambda_{j} N_{j}
$$

где $\{N\}$ - обозначение для набора чисел $N_{1}, \ldots, N_{n}$. В силу оговоренных выше свойств рассматриваемой модели для чисел $N_{j}$ выполняются условия

$$
\begin{array}{ccl}
0 \leqslant N_{j} \leqslant S_{1} \quad \text { при } & 1 \leqslant j \leqslant n_{1}, \\
0 \leqslant N_{j} \leqslant S_{2} \quad \text { при } & n_{1}+1 \leqslant j \leqslant n, \\
\sum_{j=1}^{n} N_{j}=N . &
\end{array}
$$

Финансовое осреднение расхода (86) имеет вид

$$
\mathbb{E}\left(N, S_{1}, S_{2}, \lambda, \beta\right)=-\frac{1}{\beta} \ln \left(\frac{1}{C\left(N, S_{1}, S_{2}\right)} \sum_{\{N\}}^{N, S_{1}, S_{2}} e^{-\beta \mathscr{E}(\{N\})} \prod_{j=1}^{n} \frac{1}{N_{j} !}\right),
$$

где $0<\beta-$ покупательная способность рубля, т.е. количество золота или твердой валюты, которое можно купить на один рубль,

$$
C\left(N, S_{1}, S_{2}\right)=\sum_{\{N\}}^{N, S_{1}, S_{2}} \prod_{j=1}^{n} \frac{1}{N_{j} !}
$$

а, кроме того, для суммы по всем наборам целых неотрицательных чисел $N_{1}, \ldots, N_{n}$, удовлетворяюших условиям $(87),(88)$, использовано обозначение $\sum_{\{N\}}^{N, S_{1}, S_{2}}$. 
Заметим что знак минус перед логарифмом и в экспоненте связан с тем, что покупатели стремятся минимизировать свои расходы. Сформулируем свойства финансового осреднения расхода (89).

Утверждение 3. Если $S_{1}, S_{2} \geqslant N u S_{1}^{\prime}, S_{2}^{\prime} \geqslant N$, mo

$$
\mathbb{E}\left(N, S_{1}, S_{2}, \lambda, \beta\right)=\mathbb{E}\left(N, S_{1}^{\prime}, S_{2}^{\prime}, \lambda, \beta\right),
$$

кроме того,

$$
\mathbb{E}\left(N, S_{1}, S_{2}, \lambda, \beta\right)>0 .
$$

Д о к а з а т е л ь с т в о. Для доказательства первой части утверждения достаточно заметить, что в силу условия (88) и неотрицательности чисел $N_{j}$ выполняются неравенства $N_{j} \leqslant N, j=1, \ldots, n$. Поэтому при $S_{1}, S_{2} \geqslant N$ условия (87) становятся тривиальными и не накладывают дополнительных ограничений на наборы $\{N\}$, по которым ведется суммирование в формулах (89) и (90), отсюда следует (91).

Чтобы доказать вторую часть утверждения, рассмотрим следующее неравенство:

$$
0<\frac{1}{C\left(N, S_{1}, S_{2}\right)} \sum_{\{N\}}^{N, S_{1}, S_{2}} e^{-\beta \mathscr{E}(\{N\})} \prod_{j=1}^{n} \frac{1}{N_{j} !}<e^{-\beta \lambda_{1}}<1 .
$$

Неравенство (93) является следствием (88), (90) и того, что выполняются условия $0<\beta, 0<\lambda_{1}<\lambda_{2}<\cdots<\lambda_{n}$. Подстановка (93) в (89) приводит к (92).

Рассмотрим отдельно сумму, стояшую в формуле (89) под логарифMOM:

$$
\Sigma\left(N, S_{1}, S_{2}, \lambda, \beta\right)=\sum_{\{N\}}^{N, S_{1}, S_{2}} e^{-\beta \mathscr{E}(\{N\})} \prod_{j=1}^{n} \frac{1}{N_{j} !} .
$$

Заметим, что для произвольных значений $\lambda_{j}, j=1, \ldots, n$, при $\beta=0$ сумма (94) совпадает с (90):

$$
\Sigma\left(N, S_{1}, S_{2}, \lambda, 0\right)=C\left(N, S_{1}, S_{2}\right) .
$$

Далее для удобства записи формул используем обозначение

$$
s_{j}=\left\{\begin{array}{lll}
S_{1}, & \text { если } & 1 \leqslant j \leqslant n_{1}, \\
S_{2}, & \text { если } & n_{1}+1 \leqslant j \leqslant n .
\end{array}\right.
$$

Утверждение 4. Для $\Sigma\left(N, S_{1}, S_{2}, \lambda, \beta\right)$ выполняется равенство

$$
\Sigma\left(N, S_{1}, S_{2}, \lambda, \beta\right)=\frac{A^{N}}{2 \pi} \int_{-\pi}^{\pi} d \alpha e^{-i N \alpha}\left(\prod_{j=1}^{n} \Phi_{s_{j}}\left(\frac{e^{i \alpha} e^{-\beta \lambda_{j}}}{A}\right)\right),
$$

где $A \neq 0-$ произвольное число, а Функиии $\Phi_{S}$ имеют вид

$$
\Phi_{S}(x)=\sum_{n=0}^{S} \frac{x^{n}}{n !} .
$$


Д о к а з а т е л ь с т в о. Запишем для суммы (94) следуюшее очевидное равенство:

$$
\sum_{\{N\}}^{N, S_{1}, S_{2}} e^{-\beta \mathscr{E}(\{N\})} \prod_{j=1}^{n} \frac{1}{N_{j} !}=A^{N} \sum_{N_{1}=0}^{s_{1}} \cdots \sum_{N_{n}=0}^{s_{n}} \Delta\left(\sum_{i=1}^{n} N_{i}-N\right) \prod_{j=1}^{n} \frac{e^{-\beta \lambda_{j} N_{j}}}{A^{N_{j}} N_{j} !}
$$

где $A \neq 0$ - произвольное число, $\Delta(N)$ - следующая функция целочисленного аргумента:

$$
\Delta(N)=\left\{\begin{array}{lll}
1, & \text { если } & N=0 \\
0, & \text { если } & N \neq 0
\end{array}\right.
$$

Функция (99) представляется в виде

$$
\Delta(N)=\frac{1}{2 \pi} \int_{-\pi}^{\pi} e^{i N \alpha} d \alpha
$$

Подстановка равенства (100) в формулу (98), изменение порядка суммирования и интегрирования и учет определения (97) приводят к равенству (96).

Теорема 2. Финансовое осреднение расхода (89) при заданньх значениях $N, S_{1}, S_{2}, n_{1}, \lambda_{j}, j=1, \ldots, n$, является убывающей функиией переменной $\beta$.

Док а з а т е л с т в о. Пусть $x_{k}, g_{k}, k=1, \ldots, L,-$ два набора чисел, удовлетворяющие условиям

$$
x_{k}>0, \quad \sum_{k=1}^{L} x_{k}=1 ; \quad g_{k}>0, \quad \sum_{k=1}^{L} g_{k}=1 .
$$

Рассмотрим следующие функции:

$$
\begin{aligned}
f_{1}(\beta) & =-\frac{1}{\beta} \ln \left(\sum_{k=1}^{L} g_{k} e^{-\beta x_{k}}\right) \\
f_{2}(t) & =f_{1}\left(\frac{1}{t}\right)=-t \ln \left(\sum_{k=1}^{L} g_{k} \exp \left(-\frac{x_{k}}{t}\right)\right) .
\end{aligned}
$$

Рассмотрим производную функции (103):

$$
f_{2}^{\prime}(t)=-\ln \left(\sum_{k=1}^{L} g_{k} \exp \left(-\frac{x_{k}}{t}\right)\right)-\frac{1}{t} \frac{\sum_{k=1}^{L} x_{k} g_{k} \exp \left(-x_{k} / t\right)}{\sum_{l=1}^{L} g_{l} \exp \left(-x_{l} / t\right)} .
$$

Из (101) для функции (104) следует

$$
\lim _{t \rightarrow+\infty} f_{2}^{\prime}(t)=0
$$


Также рассмотрим вторую производную:

$$
\begin{aligned}
f_{2}^{\prime \prime}(t) & =-\frac{1}{t^{3}} \frac{\sum_{k=1}^{L} x_{k}^{2} g_{k} \exp \left(-x_{k} / t\right)}{\sum_{l=1}^{L} g_{l} \exp \left(-x_{l} / t\right)}+\frac{1}{t^{3}}\left(\frac{\sum_{k=1}^{L} x_{k} g_{k} \exp \left(-x_{k} / t\right)}{\sum_{l=1}^{L} g_{l} \exp \left(-x_{l} / t\right)}\right)^{2} \\
& =-\frac{1}{t^{3}} \sum_{k=1}^{L} h_{k}\left(x_{k}-\bar{x}\right)^{2}
\end{aligned}
$$

где

$$
\begin{aligned}
h_{k} & =\frac{g_{k} \exp \left(-x_{k} / t\right)}{\sum_{l=1}^{L} g_{l} \exp \left(-x_{l} / t\right)}, \\
\bar{x} & =\sum_{k=1}^{L} h_{k} x_{k} .
\end{aligned}
$$

По определению (107) числа $h_{k}$ удовлетворяют условиям $h_{k}>0$, $\sum_{k=1}^{L} h_{k}=1$ (см. 101), поэтому из (106) следует, что при $t>0$ выполняется неравенство

$$
f_{2}^{\prime \prime}(t)<0 \text {. }
$$

Из (109) следует, что первая производная (104) является убывающей функцией при $t>0$. Отсюда в силу (105) вытекает, что при $t>0$

$$
f_{2}^{\prime}(t)>0 \text {. }
$$

Используя равенство

$$
f_{1}^{\prime}(\beta)=-\frac{1}{\beta^{2}} f_{2}^{\prime}\left(\frac{1}{\beta}\right)
$$

из (110) получим, что при $\beta>0$ выполняется неравенство

$$
f_{1}^{\prime}(\beta)<0 .
$$

Заметим теперь, что финансовое осреднение расхода (89) при любых значениях $N, S_{1}, S_{2}, n_{1}, \lambda_{j}, j=1, \ldots, n$, имеет вид (102). Отсюда в силу (112) следует утверждение теоремы.

Рассмотрим теперь финансовое осреднение расхода в пределе при $N \rightarrow \infty$. Будем считать, что $S_{1}$ и $S_{2}$ зависят от $N$ следующим образом:

$$
S_{1}=c_{1} N, \quad S_{2}=c_{2} N
$$

где $c_{1}, c_{2}$ - постоянные. Используем далее обозначение

$$
\gamma_{j}=\left\{\begin{array}{lll}
c_{1}, & \text { если } & 1 \leqslant j \leqslant n_{1}, \\
c_{2}, & \text { если } & n_{1}+1 \leqslant j \leqslant n .
\end{array}\right.
$$

Теорема 3. Пусть $c_{1}$ и $c_{2}$ таковы, что выполняются неравенства

$$
\begin{aligned}
& \frac{1-\sum_{j \notin J} \gamma_{j}}{\sum_{k \in J} e^{-\beta \lambda_{k}}} \frac{e^{-\beta \lambda_{k}}}{\gamma_{k}}<1 \quad \text { npu } \quad k \in J, \\
& \frac{1-\sum_{j \notin J} \gamma_{j}}{\sum_{k \in J} e^{-\beta \lambda_{k}}} \frac{e^{-\beta \lambda_{k}}}{\gamma_{k}}>1 \quad \text { npu } \quad k \notin J,
\end{aligned}
$$


где $J$ - подмножество множества $1, \ldots, n$. Тогда асимптотика суммь (94) при $N \rightarrow \infty$ имеет вид

$$
\begin{aligned}
& \Sigma\left(N, S_{1}, S_{2}, \lambda, \beta\right)=\frac{e^{N}}{\sqrt{2 \pi N} N^{N}} \frac{a^{N}}{\sqrt{1-\sum_{j \notin J} \gamma_{j}}} \\
& \quad \times\left(\prod_{i \notin J}\left(\frac{e^{-\beta \lambda_{i}}}{a \gamma_{i}}\right)^{N \gamma_{i}} \frac{1}{\sqrt{2 \pi N \gamma_{i}}} \frac{1}{1-a \gamma_{i} e^{\beta \lambda_{i}}}\right)\left(1+O\left(\frac{1}{N}\right)\right),
\end{aligned}
$$

¿дe

$$
a=\frac{\sum_{i \in J} e^{-\beta \lambda_{i}}}{1-\sum_{j \notin J} \gamma_{j}} .
$$

Д о к а з а т е л ь с т в о. Приведем схему доказательства. Используем равенство (96). Для этого сначала получим оценку для функций (97). Рассмотрим $\Phi_{S}(B)$ в случае, когда $S \rightarrow \infty$ и $B$ зависит от $S$ следующим образом:

$$
B=b \dot{S} \text {. }
$$

Пусть $b<1$, в этом случае

$$
\Phi_{S}(B)=e^{B}-\sum_{n=S+1}^{\infty} \frac{B^{n}}{n !} .
$$

Второе слагаемое в правой части равенства (119) представим в виде

$$
\frac{B^{S}}{S !} \sum_{k=1}^{\infty} \frac{B^{k}}{S^{k}} \prod_{i=1}^{k} \frac{1}{1+i / S}
$$

Воспользуемся следующей цепочкой неравенств:

$$
0<1-\prod_{i=1}^{k} \frac{1}{1+i / S}<1-\frac{1}{(1+k / S)^{k}}<\frac{k^{2}}{S} .
$$

Последнее неравенство в (121) является следствием того, что функция $y(x)=1-(1+x)^{-n}$ при любом $n>0$ является выпуклой вверх. Применяя (121) к (120), получим из (119) асимптотическую формулу

$$
\Phi_{S}(B)=e^{B}-\frac{B^{S}}{S !} \frac{b}{1-b}\left(1+O\left(\frac{1}{S}\right)\right) .
$$

Аналогично рассмотрим случай $b>1$. В этом случае функцию $\Phi_{S}(B)$ представим в виде

$$
\Phi_{S}(B)=\frac{B^{S}}{S !} \sum_{k=0}^{S}\left(\frac{S}{B}\right)^{k} \prod_{i=0}^{k-1}\left(1-\frac{i}{S}\right) .
$$

Воспользуемся неравенствами

$$
0<1-\prod_{i=0}^{k-1}\left(1-\frac{i}{S}\right)<1-\left(1-\frac{k-1}{S}\right)^{k-1}<\frac{(k-1)^{2}}{S}
$$


где $k>1$. Последнее неравенство в (124) является следствием того, что функция $y(x)=1-(1-x)^{k}$ при $x<1$ и $k>1$ является выпуклой вверх. Подставляя (124) в (123), получим следующую асимптотику:

$$
\Phi_{S}(B)=\frac{B^{S}}{S !} \frac{1}{1-1 / b}\left(1+O\left(\frac{1}{S}\right)\right) .
$$

Выберем в формуле (96) $A$ в виде $A=N a$ и подставим в (96) асимптотики (122) и (125), учитывая при этом (115). Получим интеграл следуюшего вида:

$$
\begin{aligned}
& \Sigma\left(N, S_{1}, S_{2}, \lambda, \beta\right) \\
& =\frac{(a N)^{N}}{2 \pi} \int_{-\pi}^{\pi} d \alpha \exp \left(-i N \alpha+i N \alpha \sum_{j \notin J} \gamma_{j}+\frac{N}{a} \sum_{j \in J} e^{-\beta \lambda_{j}} e^{i \alpha}\right) \\
& \quad \times \prod_{k \notin J} \frac{e^{-\beta \lambda_{k}}}{(N a)^{N \gamma_{k}} s_{k} !} \frac{1}{1-a \gamma_{k} e^{\beta \lambda_{k}} e^{-i \alpha}}\left(1+O\left(\frac{1}{N}\right)\right) .
\end{aligned}
$$

Функция под знаком экспоненты имеет максимум действительной части в точке $\alpha=0$, а производная ее мнимой части в этой точке равна 0 , поэтому для нахождения асимптотики интеграла (126) применим метод Лапласа. Применение метода Лапласа к интегралу (126) и учет асимптотической формулы Стирлинга для факториала приводят к выражению (116).

Из формулы (95) следует, что теорема 3 позволяет также находить асимптотику выражения (90). Подстановка асимптотики вида (116) выражений (90) и (94) в (89) приводит к тому, что асимптотика финансового осреднения расхода имеет вид:

$$
\begin{aligned}
\mathbb{E}\left(N, S_{1}, S_{2}, \lambda, \beta\right)= & N m\left(c_{1}, c_{2}, \lambda, \beta\right)+\frac{1}{2 \beta} k\left(c_{1}, c_{2}, \lambda, \beta\right) \ln (N) \\
& +l\left(c_{1}, c_{2}, \lambda, \beta\right)+O\left(\frac{1}{N}\right) .
\end{aligned}
$$

Явный вид функции $l\left(c_{1}, c_{2}, \lambda, \beta\right)$ не является существенным для дальнейшего, а функции $m\left(c_{1}, c_{2}, \lambda, \beta\right)$ и $k\left(c_{1}, c_{2}, \lambda, \beta\right)$ выражаются формулами:

$$
\begin{gathered}
m\left(c_{1}, c_{2}, \lambda, \beta\right)=m_{1}\left(c_{1}, c_{2}, \lambda, \beta\right)-m_{2}\left(c_{1}, c_{2}, \lambda, \beta\right), \\
k\left(c_{1}, c_{2}, \lambda, \beta\right)=k_{1}\left(c_{1}, c_{2}, \lambda, \beta\right)-k_{2}\left(c_{1}, c_{2}, \lambda, \beta\right),
\end{gathered}
$$

где

$$
\begin{aligned}
m_{1}\left(c_{1}, c_{2}, \lambda, \beta\right)= & -\frac{1}{\beta} \ln a+c_{1} \sum_{i=1}^{p_{1}} \lambda_{i}+\frac{p_{1} c_{1}}{\beta} \ln \left(a c_{1}\right) \\
& +c_{2} \sum_{i=n_{1}+1}^{p_{2}} \lambda_{i}+\frac{\left(p_{2}-n_{1}\right) c_{2}}{\beta} \ln \left(a c_{2}\right), \\
k_{1}\left(c_{1}, c_{2}, \lambda, \beta\right)= & p_{1}+p_{2}-n_{1}
\end{aligned}
$$


и

$$
a=\frac{1}{1-c_{1} p_{1}-c_{2}\left(p_{2}-n_{1}\right)}\left(\sum_{i=p_{1}+1}^{n_{1}} e^{-\beta \lambda_{i}}+\sum_{i=p_{2}+1}^{n} e^{-\beta \lambda_{i}}\right) .
$$

В формулах (128)-(131) $p_{1}$ и $p_{2}$ - целые числа, которые зависят от $c_{1}, c_{2}, \lambda, \beta$ и однозначно определяются из следующих условий:

$$
\begin{array}{rlrl}
1 & \leqslant p_{1} \leqslant n_{1}, & e^{-\beta \lambda_{p_{1}+1}}<a c_{1}<e^{-\beta \lambda_{p_{1}}}, \\
n_{1}+1 \leqslant p_{2} \leqslant n, & e^{-\beta \lambda_{p_{2}+1}}<a c_{2}<e^{-\beta \lambda_{p_{2}}} .
\end{array}
$$

Вид функций $m_{2}\left(c_{1}, c_{2}, \lambda, \beta\right)$ и $k_{2}\left(c_{1}, c_{2}, \lambda, \beta\right)$ зависит от значений $c_{1}$ и $c_{2}$ следуюшим образом. Если выполняются условия

$$
c_{1}>n^{-1}, \quad c_{2}>n^{-1}
$$

To

$$
m_{2}\left(c_{1}, c_{2}, \lambda, \beta\right)=-\frac{1}{\beta} \ln (n), \quad k_{2}\left(c_{1}, c_{2}, \lambda, \beta\right)=0 .
$$

Если выполняется

$$
c_{1}>n^{-1}, \quad c_{2}<n^{-1}
$$

To

$$
m_{2}\left(c_{1}, c_{2}, \lambda, \beta\right)=-\frac{1}{\beta} \ln (b)+\frac{n_{2} c_{2}}{\beta} \ln \left(b c_{2}\right), \quad k_{2}\left(c_{1}, c_{2}, \lambda, \beta\right)=n_{2},
$$

где

$$
b=\frac{n_{1}}{1-c_{2} n_{2}} .
$$

В случае, когда выполняются условия

$$
c_{1}<n^{-1}, \quad c_{2}>n^{-1},
$$

функции $m_{2}$ и $k_{2}$ выражаются формулами

$$
m_{2}\left(c_{1}, c_{2}, \lambda, \beta\right)=-\frac{1}{\beta} \ln (b)+\frac{n_{1} c_{1}}{\beta} \ln \left(b c_{1}\right), \quad k_{2}\left(c_{1}, c_{2}, \lambda, \beta\right)=n_{1},
$$

где

$$
b=\frac{n_{2}}{1-c_{1} n_{1}} .
$$

Из теоремы 2 следует, что $m\left(c_{1}, c_{2}, \lambda, \beta\right)$ при фиксированных $c_{1}, c_{2}, \lambda$ является убывающей функцией переменной $\beta$. Этот факт имеет простую экономическую интерпретацию: если покупательная способность рубля $\beta$ растет, то покупатель тратит на покупку меньшее количество рублей.

Введем теперь понятия расхода одного человека и скорости оборота. Расходом одного человека будем называть следующее выражение:

$$
M\left(N, S_{1}, S_{2}, \lambda, \beta\right)=\frac{1}{2}\left(\mathbb{E}\left(N+1, S_{1}, S_{2}, \lambda, \beta\right)-\mathbb{E}\left(N-1, S_{1}, S_{2}, \lambda, \beta\right)\right) .
$$


Скоростью оборота будем называть выражение

$$
V\left(N, S_{1}, S_{2}, \lambda, \beta\right)=\frac{\partial}{\partial N} M\left(N, S_{1}, S_{2}, \lambda, \beta\right) .
$$

Из (127) следует, что расход одного человека при $N \rightarrow \infty$ имеет вид

$$
M\left(N, S_{1}, S_{2}, \lambda, \beta\right)=m\left(c_{1}, c_{2}, \lambda, \beta\right)+\frac{1}{2 \beta N} k\left(c_{1}, c_{2}, \lambda, \beta\right)+O\left(\frac{1}{N^{2}}\right),
$$

а скорость оборота есть

$$
V\left(N, S_{1}, S_{2}, \lambda, \beta\right)=-\frac{1}{2 \beta N^{2}} k\left(c_{1}, c_{2}, \lambda, \beta\right)+O\left(\frac{1}{N^{3}}\right) .
$$

В общем случае выражение (133) слишком сложное для аналитического исследования. Рассмотрим случай, когда $n_{2}=n_{1}=n / 2$. В этом случае из (133) следует, что максимальное значение главного члена асимптотики скорости оборота равно

$$
V\left(N, S_{1}, S_{2}, \lambda, \beta\right)=-\frac{n}{4 \beta N^{2}}
$$

и достигается при

$$
c_{1}=\frac{e^{-\beta \lambda_{1}}}{\sum_{j=1}^{n} e^{-\beta \lambda_{j}}}, \quad c_{2}=\frac{e^{-\beta \lambda_{n_{1}+1}}}{\sum_{j=1}^{n} e^{-\beta \lambda_{j}}} .
$$

Из (135) следует, что одним из условий максимальности скорости оборота является следуюшее: число дешевых товаров должно быть больше, чем число дорогих.

Далее покупателя акций будем называть игроком. Пусть существует всего два типа акций, первый условно будем называть «дешевыми» акциями, а второй - «дорогими». Будем считать, что игрок покупает пакет из $N$ акций, в котором количество дешевых акций равно $N_{1}$, а количество дорогих акций соответственно равно $N_{2}=$ $N-N_{1}$. Игрок тратит деньги на покупку акций, при этом покупаемое количество акций оказывает влияние на цену акций обоих типов. В частности, чем больше дорогих акций покупает игрок, тем дешевле их ему продадут. Поэтому далее будем считать, что расход игрока на пакет акций зависит от количества купленных дешевых и дорогих акций нелинейно. Например, квадратичным образом:

$$
\begin{aligned}
\mathscr{E}\left(N_{1}\right) & =\lambda_{1} N_{1}+\lambda_{2} N_{2}-\frac{\gamma N_{1}^{2}}{2 N}-\frac{\gamma N_{2}^{2}}{2 \dot{N}} \\
& =\lambda_{2} N-\frac{\gamma N}{2}+\left(\lambda_{1}-\lambda_{2}+\gamma\right) N_{1}-\frac{\gamma N_{1}^{2}}{2 N},
\end{aligned}
$$

где числа $\lambda_{1}, \lambda_{2}, \gamma$ удовлетворяют условиям

$$
\lambda_{1}<\lambda_{2}, \quad \lambda_{2}-\lambda_{1}<\gamma .
$$


Из условий (137) следует, что функция (136) при $N_{1}=0,1, \ldots, N$ имеет глобальный минимум при $N_{1}=N$ и локальный минимум при $N_{1}=0$. Это означает, что хотя расход покупателя минимален, если он купил только дешевые акции, однако купить только дорогие акции тоже в некотором смысле выгодно.

Рассмотрим теперь локальные финансовые осреднения дохода игрока. Будем считать, что дешевые акции игрок может купить у $G_{1}$ дилеров, а дорогие - у $G_{2}$ дилеров. В таком случае количество разных способов, которым игрок может купить пакет акций, равно

$$
\Gamma\left(N_{1}\right)=\frac{\left(N_{1}+G_{1}-1\right) !}{\left(G_{1}-1\right) ! N_{1} !} \frac{\left(N-N_{1}+G_{2}-1\right) !}{\left(G_{2}-1\right) !\left(N-N_{1}\right) !} .
$$

3 а м е ч а н и е. Вместо введения разных дилеров можно было бы считать, что самих дешевых и дорогих акций соответственно $G_{1}$ и $G_{2}$ разных типов, которые стоят одинаково.

Кроме того, будем считать, что игрок действует следующим образом: в начальный момент времени игрок покупает некоторый пакет акций, а затем на каждом временном шаге он продает одну акцию одного типа и покупает одну акцию другого типа так, чтобы его расход при этом уменьшился. То есть игрок не рассматривает перспективу, а на каждом шаге действует так, чтобы минимизировать свой расход. Локальное финансовое осреднение расхода игрока в таком случае равно

$$
\mathbb{E}\left(N_{1}^{0}, \beta\right)=-\ln \left(\sum_{N_{1} \in I\left(N_{1}^{0}\right)} \Gamma\left(N_{1}\right) e^{-\beta \mathscr{E}\left(N_{1}\right)}\right)=-\ln \left(\sum_{N_{1} \in I\left(N_{1}^{0}\right)} e^{-\beta F\left(N_{1}, \beta\right)}\right)
$$

где $\beta$ - покупательная способность рубля, функция $F\left(N_{1}, \beta\right)$ выражается формулой

$$
F\left(N_{1}, \beta\right)=\mathscr{\delta}\left(N_{1}\right)-\frac{1}{\beta} \ln \left(\Gamma\left(N_{1}\right)\right),
$$

а $I\left(N_{1}^{0}\right)$ обозначает множество, образованное набором чисел $N_{1 a}$, $a=0,1, \ldots, A$, таких, что выполняются условия

$$
\begin{gathered}
\left|N_{1 a}-N_{1, a+1}\right|=1, \quad F\left(N_{1, a+1}, \beta\right)<F\left(N_{1 a}, \beta\right), \quad a=0,1, \ldots, A-1, \\
F\left(N_{1 A}\right)<F\left(N_{1 A} \pm 1\right) \quad \text { и } \quad N_{10}=N_{1}^{0} .
\end{gathered}
$$

Рассмотрим локальные финансовые осреднения (139) в пределе при $N \rightarrow \infty$. Будем при этом считать, что $G_{1}$ и $G_{2}$ зависят от $N$ так, что

$$
\lim _{N_{1} \rightarrow \infty} \frac{G_{1}}{N}=g_{1}>0, \quad \lim _{N_{1} \rightarrow \infty} \frac{G_{2}}{N}=g_{2}>0 .
$$

В таком случае асимптотика локальных финансовых осреднений расхода (139) определяется минимальными значениями следующей 
функции:

$$
\begin{aligned}
f(n, \beta)= & \lambda_{2}-\frac{\gamma}{2}+\left(\lambda_{1}-\lambda_{2}+\gamma\right) n-\frac{\gamma n^{2}}{2} \\
- & \frac{1}{\beta}\left(\left(g_{1}+n\right) \ln \left(1+\frac{n}{g_{1}}\right)-n \ln \left(\frac{n}{g_{1}}\right)\right. \\
& \left.\quad+\left(g_{2}+1-n\right) \ln \left(1+\frac{1-n}{g_{2}}\right)-(1-n) \ln \left(\frac{1-n}{g_{2}}\right)\right),
\end{aligned}
$$

где $n$ принимает значения на отрезке $[0,1]$. Оказывается, что существует критическое значение $\beta_{c}\left(\lambda_{1}, \lambda_{2}, \gamma, g_{1}, g_{2}\right)$ покупательной способности рубля. При $\beta>\beta_{c}$ функция (140) имеет на отрезке $n \in[0,1]$ два минимума - глобальный и локальный, - a, кроме того, между этими минимумами находится точка максимума. Пусть минимумы функции (140) находятся в точках $n_{1}(\beta)$ и $n_{2}(\beta)$, а максимум - в точке $n_{3}(\beta)$, причем $n_{1}<n_{3}<n_{2}$. Справедливо следующее утверждение.

Утверждение 5. При $N \rightarrow \infty u \beta>\beta_{c}$ все значения $N_{1}^{0}$ делятся на две части таким образом, что:

$$
\begin{array}{lll}
\frac{1}{N} \mathbb{E}\left(N_{1}^{0}, \beta\right) \approx f\left(n_{1}(\beta), \beta\right) & n p u & N_{1}^{0}<N n_{3}(\beta), \\
\frac{1}{N} \mathbb{E}\left(N_{1}^{0}, \beta\right) \approx f\left(n_{2}(\beta), \beta\right) & n p u & N_{1}^{0}>N n_{3}(\beta) .
\end{array}
$$

При $\beta \rightarrow \beta_{c}+0$ локальный минимум и максимум сливаются и при $\beta<\beta_{c}$ перестают существовать. Таким образом, если покупательная способность рубля меньше критической, функция (140) имеет на отрезке $[0,1]$ только одну точку экстремума - глобальный минимум. Обозначим эту точку через $n_{0}(\beta)$.

Утверждение 6. При $N \rightarrow \infty u \beta<\beta_{c}$ для всех значений $N_{1}^{0}$ вьгполняется

$$
\frac{1}{N} \mathbb{E}\left(N_{1}^{0}, \beta\right) \approx f\left(n_{0}(\beta), \beta\right) \text {. }
$$

Исчезновение локального минимума при уменьшении покупательной способности рубля можно интерпретировать как «пробой акций». А именно, игрок, который в результате своей стратегии получения выигрыша на каждом шаге оказался в локальном минимуме, при достижении критического значения покупательной способности рубля уже больше не находится в точке минимума расходов, и поэтому из стремления к выгоде такой игрок должен совершить скачек в точку глобального минимума.

Аналогично можно рассмотреть случай $N$ покупателей, которые покупают два разных товара. При этом покупатели изменяют количество покупаемого товара, чтобы минимизировать расход, но делают это медленно, из-за чего при уменьшении покупательной стоимости рубля происходит дефолт. 
В этом случае изменятся кратности (138), которые уже будут иметь не бозонный, а больцмановский вид:

$$
\Gamma\left(N_{1}\right)=N ! \frac{G_{1}^{N_{1}}}{N_{1} !} \frac{G_{2}^{N-N_{1}}}{\left(N-N_{1}\right) !} .
$$

Соответственно изменится и функция (140), в больцмановском случае она примет вид

$$
\begin{aligned}
f(n, \beta)= & \lambda_{2}-\frac{\gamma}{2}+\left(\lambda_{1}-\lambda_{2}+\gamma\right) n-\frac{\gamma n^{2}}{2} \\
& +\frac{1}{\beta}\left(n \ln \left(\frac{n}{g_{1}}\right)+(1-n) \ln \left(\frac{1-n}{g_{2}}\right)\right) .
\end{aligned}
$$

Все остальное сказанное выше относительно критической покупательной способности и локальных финансовых осреднений остается в силе. Зависимость точек экстремума функции (141) от покупательной способности рубля можно интерпретировать как лагранжево многообразие

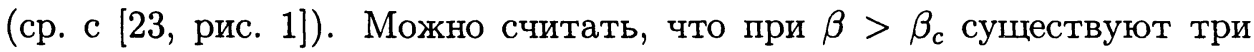
ветви лагранжева многообразия: $n_{1}(\beta), n_{2}(\beta)$ и $n_{3}(\beta)$. При $\beta=\beta_{c}$ две из этих ветвей приходят в одну точку и при $\beta>\beta_{c}$ перестают существовать, остается только одна ветвь $n_{0}(\beta)$. В силу свойств функции (141) проекция двух сливающихся ветвей и проекция третьей ветви на ось $n$ не пересекаются ни в одной точке. Это означает, что при достижении критической покупательной способности происходит дефолт. Так как проекции на ось $n$ разных ветвей лагранжева многообразия не пересекаются, то покупатели не могут за счет малого изменения количества покупаемого товара изменить покупательную способность рубля и перейти с одной ветви на другую. Поэтому при достижении критического значения покупательной способности рубля исчезновение ветвей лагранжева многообразия означает исчезновение состояния равновесия для покупателей, находившихся на этих ветвях.

В заключение хочу выразить глубокую благодарность В.Н. Батурину и С.Г. Лебедеву, которые инициировали реанимацию моих работ 1987-1990 годов (см. [24]), приведших к правильному прогнозированию сегодняшней экономической ситуации в стране, а также Г. В. Ковалю за помощь в подготовке данной работы.

\section{СПИСОК ЛИТЕРАТУРЫ}

1. Вьюгин В. В., Маслов В. П. Об экстремальных соотношениях между аддитивными функциями потерь и колмогоровской сложностью. - Проблемы передачи информации, 2003 , т. 39, № 4, с. 71-87.

2. Маслов $B . \Pi$., Черныцй $A . C$. О минимизации и максимизации энтропии в различных дисциплинах. - Теория вероятн. и ее примен., 2003 , т. 48, в. 3, с. 466-486. 
3. Маслов В. П. Замечание о компьютерно-ориентированном языке. - Проблемы передачи информации, 2003, т. 39, № 3, с. 72-76.

4. Ширяев А.Н. Вероятность. М.: Наука, 1989, 640 с.

5. Маслов В. П. Аксиомы нелинейного осреднения в финансовой математике и аналог фазового перехода. - Докл. РАН, 2003, т. 393, № 6, с. 735-739.

6. Маслов В.П. Аксиомы нелинейного осреднения в финансовой математике и динамика курса акций. - Теория вероятн. и ее примен., 2003, т. 48, в. 4, с. 799-810.

7. Maslov V.P. The notions of entropy, Hamiltonian, temperature, and thermodynamical limit in probability theory used for solving model problems in econophysics. Russian J. Math. Phys., 2002, v. 9, № 4, p. 437-445.

8. Маслов В. П. Операторные методы. М.: Наука, 1973, 544 с.

9. Федорюк М. В. Метод перевала. М.: Наука, 1977, 368 с.

10. Маслов В.П. Зависимость покупательной способности и среднего дохода населения от числа покупателей на специализированном рынке и в регионе. Законы эконофизики. - Докл. РАН, 2004, т. 395, № 2, с. 164-168.

11. Маслов В. П. Расходы покупателей и скорость оборота при нелинейном финансовом осреднении. Законы эконофизики. - Докл. РАН, 2004, т. 396, № 2 (в печати).

12. Dragulescu A., Yakovenko V.M. Exponential and power-law probability distributions of wealth and income in United Kingdom and the United States. - Phys. A, 2001, v. 299 , p. 213-221.

13. Чеботарев A.M. Распределение Парето как результат компьютерной реконструкции статистики авторынка России. - Укр. журн. «Економіст», 2003, № 7, c. $6-9$.

14. Лошинин $M$. Закон Парето: потребность переоткрытия. - Укр. журнал «Економіст», 2003, № 2, с. 58-68.

15. Reed W. J. The Pareto law of incomes - an explanation and an extension. - Phys. A, 2003, v. 319, № 1-4, p. 469-486.

16. Nagahara Yu., Okazaki M. Pareto's law for income of individuals and debt of bankrupt companies Hideaki Aoyama and Wataru Souma. - Fractals, 2000, v. 8, № 3, p. 293300.

17. Sornette D., Zajdenweber D. Economic returns of research: the Pareto law and its implications. - European Phys. J. B, 1999, v. 8, p. 653-664.

18. Dagum C. A systemic approach to the generation of income distribution models. J. Income Distribution, 1996, v. 6, № 1, p. 105-126.

19. Pareto $V$. Cours d'économie politique. V. 2. Paris: F. Pichou, 1897.

20. Маслов В.П. Интегральные уравнения и фазовые переходы в вероятностных играх. Аналогия со статистической физикой. - Теория вероятн. и ее примен., 2003 , т. 48 , в. 2 , с. $403-411$.

21. Маслов В. П. Аппроксимационные вероятности, закон квазистабильного рынка и фазовый переход из «конденсатного» состояния. - Докл. РАН, 2003, т. 392, № 6, c. $727-732$.

22. Маслов В. П. Среднестатистическая информация относительно выпуклой функции и общие линейные уравнения для пространства $\min , \max$. - Докл. PAH, 2003 , т. 393 , № 1 , с. $20-24$.

23. Маслов В.П. Об одной модели высокотемпературной сверхпроводимости. Докл. РАН, 2003, т. 391, № 5, с. 605-609.

24. Маслов В. П. Экспертизы и эксперименты. - Новый мир, 1991, № 1, с. 243-252.

Поступила в редакцию 23.IV.2004 